\title{
O IMPACTO DA RESPONSABILIDADE SOCIAL NA SATISFAÇÃO DOS TRABALHADORES
}

\author{
Macário Neri Ferreira Neto ${ }^{1}$ \\ Annara Cristina Oliveira Santos ${ }^{2}$ \\ Randal Martins Pompeu ${ }^{3}$
}

\section{RESUMO}

A responsabilidade social corporativa se aplica a diferentes questões e perspectivas, como ética nos negócios, contrato social, sustentabilidade ambiental e cidadania corporativa. Nesse contexto e com base na teoria dos stakeholders, este trabalho tem como objetivo verificar se a responsabilidade social percebida pelo empregado influencia sua satisfação no trabalho, verificando também o efeito mediador da significância do traba lho e do suporte organizacional percebido como potencia liza dores dessa relação. A amostra contou com 190 empregad os de uma instituição de ensino que responderam a um questionário on-line, disponibilizados por intermédio da universidade. Para análise dos dados, esta pesquisa utilizou análises descritivas e modelagem de equações estruturais. Os resultados do estudo indicam que a responsabilidade social corporativa percebida influencia a satisfação do trabalhador, quando mediada pela significância do trabalho e/ou pelo suporte organizacional percebido. Quando a responsabilida de social percebida foi testada, diretamente, com a satisfação do trabalhador, o resultado não se mostrou significativo. Os resultados desta pesquisa geram implicações efetivas para as organizações ao demonstrar que as ações de responsabilidade social podem gerar benefícios relevantes internamente, a lém dos próprios benefícios gerados para a sociedade.

Palavras-chave: Responsabilidade social percebida. Satisfação no trabalho. Significância do trabalho. Suporte organizacional percebido.

\section{THE IMPACT OF SOCIAL RESPONSIBILITY ON EMPLOYEE SATISFACTION}

\begin{abstract}
Corporate social responsibility applies to different issues and perspectives, such as business ethics, social contract, environmental sustainability and corporate citizenship. In this context and based on the stakeholder theory, this work aims to verify whether the employees' perceived social responsibility influences their job satisfaction, also verifying the mediating effect of the significance of work and organizationalsupport perceived as enhancing this relationship. The sample consisted of 190 employees of an educational institution who answered an online questionnaire, made available through the university. For data analysis, this re search used descriptive analysis and structural equation modeling. The study results indicate that perceived corporate social responsibility influences worker satisfaction when mediated by the significance of work and/or perceived organizational support. When perceived social responsibility was directly tested with worker satisfaction, the result was not significant. The results of this research generate effective implications for organizations by demonstrating that social responsibility actions can generate relevant benefits internally, in addition to the benefits generated for society.
\end{abstract}

Keywords: Perceived social responsibility. Job satisfaction. Significance of work. Perceived organizational support.

\footnotetext{
1 Doutorando em Administração de Empresas pela Universidade de Fortaleza, Ceará (Brasil). Email: macario ferreira@hotmail.com Orcid: https://orcid.org/0000-0002-9697-4066

${ }^{2}$ Graduação em Administração pela Universidade Federal do Ceará, Ceará (Brasil). Atualmente é Administrador do Instituto Federal do Ceará - Reitoria. Email: annara.santos@edu.unifor.br Orcid: https://orcid.org/0000$\underline{0002-1621-5554}$
}

${ }^{3}$ Doutor em Gestão pela Universidade de Trás-os-Montes e Alto Douro (UTAD- Portugal). Vice-Reitor de Extensão e Comunidade Universitária e Professor Titular do Programa de Pós-Graduação em Administração de Empresas (PPGA) da Universidade de Fortaleza (UNIFOR), Ceará (Brasil). Email: randal@unifor.br Orcid: https://orcid.org/0000-0003-4154-3725 


\section{INTRODUÇÃO}

O ambiente de trabalho, repleto de incertezas, contribui para a falta de confiança e de preocupação com o bem-estar mútuo entre empregados e empregadores. Muitos empregadores ainda não compreenderam totalmente a importância central de ter relacionamentos favoráveis com os empregados para reduzir o absenteísmo, bem como aumentar a dedicação aos objetivos organizacionais e aumentar o desempenho (Eisenberger, Malone, \& Presson, 2016; Glavas \& Kelley, 2014; Nazir \& Islam, 2020).

Nesse sentido, parte da literatura mostra que a participação de qualquer empresa em ações de responsabilidade social corporativa proporciona impacto positivo nas atitudes de trabalho e nos comportamentos das partes interessadas internas e externas (Khan, Sarwar, \& Khan, 2018).

Portanto, a percepção da responsabilidade social promove maior satisfação e comprometimento no trabalho, melhora a performance do trabalhador e traz significância, segurança e disponibilidade dos empregados, identificação com a empresa e envolvimento com o trabalho (Chaudhary, 2019; Hur, Moon, \& Choi, 2021; Khan et al., 2018).

Estudos anteriores buscaram avaliar a relação entre a responsabilidade social corporativa (RSC) percebida e a satisfação com o trabalho antecedidas por programas de ética (Valentine \& Fleischman, 2007): a RSC percebida como antecedente do compromisso organizacional e satisfação no trabalho (Asrar-ul-Haq, Kuchinke, \& Iqbal, 2017); a RSC percebida e satisfação no trabalho mediados pela identificação organizacional (Boğan, Türkay, \& Dedeoğlu, 2018; Tamm, Eamets, \& Mõtsmees, 2010); e a RSC percebida como antecedente do engajamento e significância do trabalho (Afsar, Al-Ghazali, \& Umrani, 2020).

No entanto, não foram localizados trabalhos que utilizem a significância do trabalho e o suporte organizacional percebido como partes integrantes de um mesmo modelo, ou seja, juntamente com RSC e satisfação do trabalhador. Ademais, estudos que incluam a significância do trabalho ainda são pouco explorados (Glavas \& Kelley, 2014). Assim, este trabalho difere dos anteriores pelo modelo teórico utilizado e pelo uso da técnica de equações estruturais, análise multigrupo e validação de uma escala de RSC percebida.

Diante do exposto, surgiu a seguinte questão: a responsabilidade social corporativa percebida influencia a satisfação do trabalhador diretamente ou quando mediada pela significância do trabalho e/ou pelo suporte organizacional percebido?

Assim, o objetivo deste trabalho é verificar se a responsabilidade social corporativa percebida pelo empregado influencia sua satisfação no trabalho, verificando também o efeito mediador da significância do trabalho e do suporte organizacional percebido como potencializadores dessa relação, além de verificar as diferenças entre os trabalhadores do sexo masculino e feminino.

A pesquisa utilizou questionários aplicados aos empregados de uma instituição de ensino superior privada, localizada em Fortaleza - CE. Os dados coletados foram tratados estatisticamente e as hipóteses foram verificadas com uso de equações estruturais.

A relevância da pesquisa está na contribuição da responsabilidade social corporativa na satisfação do trabalhador, podendo gerar benefícios relevantes não somente para a sociedade de uma forma geral, mas também para os empregados das organizações, bem como no avanço do entendimento da relação entre RSC percebida e indicadores importantes para a área de recursos humanos.

Para o mercado, espera-se contribuir com a discussão da relevância da RSC das empresas e seus impactos positivos junto aos trabalhadores. Os resultados desta pesquisa devem ser benéficos para as organizações que operam no Brasil, permitindo-lhes determinar 
onde é melhor concentrar suas atividades de RSC para maximizar o comprometimento de seus funcionários.

\section{REFERENCIAL TEÓRICO}

Nesta seção é apresentada a base teórica dos construtos que embasam o modelo teórico proposto pelo trabalho. A seção está dividido em responsabilidade social corporativa percebida, significância do trabalho, suporte organizacional percebido e satisfação do trabalhador.

\subsection{Responsabilidade social corporativa percebida}

Com contribuições marcantes para o desenvolvimento da responsabilidade social corporativa (RSC) nos aspectos modernos, Carroll (1979) apontou a responsabilidade social das organizações em uma estrutura piramidal: econômica, jurídica, ética e filantrópica. De acordo com essa pirâmide, a responsabilidade fundamental das organizações é a responsabilidade econômica, que se refere à produção e venda de produtos para obter lucro como uma organização comercial. Enquanto a responsabilidade legal se refere ao dever da organização de seguir os regulamentos legais durante a realização de suas atividades, a responsabilidade ética se refere a agir de acordo com as normas e valores não incluídos nos regulamentos legais, mas geralmente aceitos pela sociedade. Por fim, a responsabilidade filantrópica abrange as responsabilidades conscienciosas da organização (Carroll, 2016).

A RSC pode ser entendida como práticas ou atividades organizacionais que satisfazem várias obrigações das empresas para uma variedade de partes interessadas, incluindo acionistas, clientes, empregados, comunid ades locais e meio ambiente, no que diz respeito a todos os procedimentos das atividades de negócios (Carroll, 1999; Kim, Nurunnabi, Kim, \& Jung, 2018b; Rowley \& Berman, 2000).

Aguinis (2011) define atividades de RSC como ações e políticas organizacionais específicas ao contexto, que levam em consideração as expectativas das partes interessadas e o triplo resultad o final do desempenho econômico, social e ambiental.

Entretanto, tem havido uma visão divergente sobre se a RSC é uma responsabilidade do acionista ou uma obrigação social. A RSC como obrigação social é explicada por meio da teoria dos stakeholders, que preconiza que uma corporação, assim como seus acionistas, também deve levar em consideração os interesses de outros stakeholders. A teoria do contrato social acentua isso e afirma que, como a sociedade fornece às empresas os recursos para operar, as empresas devem aderir às normas e aos valores sociais em troca, além de contribuir para a comunidade por meio de vários programas de bem-estar social (Ahmad, Shafique, \& Jamal, 2020).

Assim, a RSC inclui as atividades que uma empresa realiza tentando afetar positivamente a sociedade e o meio ambiente (Afsar et al., 2020). Nesse sentido, as instituições de ensino superior constituem-se como espaços para a produção acadêmica e formação profissional e, apesar da centralização no tripé ensino, pesquisa e extensão, elas devem buscar maneiras de contribuir para solucionar os problemas da sociedade (Lima, Ferreira Neto, \& Pompeu, 2020).

Os indivíduos, como seres sociais, mantêm suas vidas em interação com os outros e, conforme as informações derivadas de ideologias, costumes e tradições religiosas, as pessoas podem desejar que os outros se comportem da mesma maneira e, portanto, podem identificar as injustiças para com as pessoas ao seu redor como sendo com elas mesmas (Boğan et al., 2018). 
Através do desejo de sustentar uma identidade positiva para sua organização e para si próprios (identidade esta desencadeada pelas ativid ades de RSC da empresa), os trabalhad ores executam suas funções com mais disposição e melhoram seu desempenho no trabalho (Hur et al., 2019). Além disso, por meio da implementação de iniciativas de RSC, as organizações podem melhorar a reputação organizacional para enriquecer a satisfação dos empregados no trabalho (Priyanka, Thevanes, \& Arulrajah, 2020).

Assim, tem-se a responsabilidade social corporativa percebida, definida como a percepção que os stakeholders de uma organização têm sobre o impacto das estratégias e práticas operacionais desta empresa no bem-estar de todas as principais partes interessadas e no ambiente natural (Glavas \& Godwin, 2013).

Para Ahmad et al. (2017), a percepção da RSC representa a cognição individual sobre a importância da conformidade com a RSC e, também, a percepção dos empregados sobre os programas de RSC organizacionais. Para esses autores, tanto para a pesquisa quanto para a prática, é necessário avaliar o impacto psicológico das atividades de RSC pelo empregador a partir da perspectiva dos empregados.

As organizações, quando atuam como modelos e cidadãos globais, voltadas para a elevação da sociedade e o cuidado com o meio ambiente e genuinamente tentando implementar políticas de RSC, fazem os empregados se sentirem responsáveis, e eles também tendem a mostrar a intenção de se engajarem em comportamentos que podem tornar a vida melhor para todos (Afsar et al., 2020).

\subsection{Significância do trabalho}

A significância do trabalho se refere ao se sentir útil e valorizado pela organização (Afsar et al., 2020), sendo definida como a percepção de fazer um trabalho significativo, que permite às pessoas expressarem seu potencial e alcançarem seu propósito (Ghislieri, Cortese, Molino, \& Gatt, 2019).

Uma pessoa se sente significativa se puder ser útil e valiosa para sua organização (Purba, Chaterine, Hardjono, \& Clarissa, 2019). Assim, o sentido de propósito e realização prevalece entre os colaboradores, que percebem fazer parte de uma organização que se comporta de forma socialmente responsável e que implementa programas que visam a contribuir para a melhoria da sociedade e do meio ambiente (Afsar et al., 2020).

Embora a percepção da RSC influencie positivamente a significância do trabalho, a extensão em que a significância do trabalho pode ser obtida varia dependendo das percepções do indivíduo sobre a consistência do objetivo e suas expectativas (Chaudhary \& Akhouri, 2019). Nesse sentido, os programas de RSC que tentam abordar genuinamente as questões ambientais e sociais podem aumentar a autoestima do empregado, o que, por sua vez, aumenta o sentimento de significância no trabalho (Aguinis \& Glavas, 2019).

Portanto, o bom desempenho de RSC transmite a mensagem de que os empregados podem servir aos outros e à sociedade, mais do que apenas ganhar a vida trabalhando em determinada organização, o que ajuda os empregados a identificar significados em seu trabalho (Azim, 2016).

Em outras palavras, a importância do trabalho percebida pelos empregados e sua motivação intrínseca indicam a eficácia da implementação da RSC. Ao contrário, se os empregados não percebem a significância do trabalho, apesar dos esforços para implementar práticas de RSC, estas práticas são consideradas menos eficazes na organização (Kim, Chang, \& Kim, 2018a).

A sensação de pertencer a algo especial faz com que os funcionários experimentem maior significado psicológico (Chaudhary, 2019). Para Grant (2008), a percepção de valor 
social e impacto social afetará o senso de propósito e de valor dos funcionários. A participação no plano de responsabilidade social corporativa da organização traz mais significado à vida dos colaboradores e oportunidades de fazer contribuições importantes para a sociedade.

Em pesquisa feita na Coreia do Sul, com a participação de 378 empregados de empresas de grande, médio e pequeno porte, com dados coletados por meio de uma pesquisa on-line, Kim et al. (2018b) indicaram que a percepção da RSC está positivamente associada com a significância do trabalho e com o suporte organizacional percebido. Afsar et al. (2020) e Nazir e Islam (2020), em seus estudos, verificaram a existência de relação positiva entre RSC e significância do trabalho. Portanto, de acordo com essas descobertas, tem-se a seguinte hipótese:

\section{H1 - A responsabilidade social percebida impacta a significância do trabalho.}

\subsection{Suporte organizacional percebido (SOP)}

Conforme Eisenberger et al. (2016), é fundamental que as empresas reconheçam os empregados como fontes valiosas de capital humano, para o benefício tanto dos empregados quanto das próprias organizações. Nesse contexto, o suporte organizacional percebido (SOP), que significa a percepção do empregado de que a organização valoriza suas contribuições de trabalho e se preocupa com seu bem-estar, demonstrou ter benefícios importantes para empregados e empregad ores (Eisenberger et al., 2016).

Por exemplo, estudos descobriram que empregados com alto suporte organizacional percebido (SOP) sofrem menos estresse no trabalho e estão mais inclinados a retornarem ao trabalho mais cedo após um acidente (Shaw et al., 2013). Além disso, o alto SOP está positivamente relacionado ao desempenho (Kurtessis et al., 2015; Rhoades \& Eisenberger, 2002).

A teoria do suporte organizacional explica como o suporte organizacional percebido (SOP) desenvolve e produz consequências positivas para empregados e organizações. A teoria afirma que os empregados veem sua organização como tendo uma disposição para vê-los de forma favorável ou desfavorável, conforme refletido no tratamento que lhes oferece (Eisenberger et al., 1986).

Ademais, a teoria do suporte organizacional tem atraído a atenção de estudiosos e profissionais da área empresarial, uma vez que o conceito é conhecido por estar fortemente relacionado às várias percepções, atitudes e comportamentos críticos, tais como compromisso organizacional afetivo, identificação organizacional, envolvimento no trabalho, satisfação no trabalho, confiança, rotativid ade, organização, comportamento de cidadania e desempenho na carreira (Kim et al., 2018a).

Assim, o suporte organizacional percebido (SOP) seria valorizado pelos empregados por atender às necessidades socioemocionais, fornecendo a indicação da prontidão da organização para recompensar o aumento do esforço de trabalho e apontando a inclinação da organização para fornecer ajuda, quando necessário, de forma que o empregado realize seu trabalho de forma eficaz (Eisenberger et al., 1986).

De acordo com Ellis (2009), as percepções dos empregados sobre as políticas de RSC de uma empresa estão positivamente relacionadas ao suporte organizacional percebido por eles. Diante disso, as empresas com bom desempenho social tendem a tratar todos os stakeholders de forma mais positiva, especialmente um dos mais importantes stakeholders os empregados. Portanto, a percepção dos empregados sobre a RSC levará à percepção do suporte organizacional (Wang, 2018). Conforme a teoria apresentada, tem-se a seguinte hipótese: 


\author{
H2 - A responsabilidade social percebida impacta o suporte organizacional \\ percebido.
}

\title{
2.4 Satisfação no trabalho
}

Segundo Aziri (2011), a satisfação no trabalho representa um sentimento que surge como resultado da percepção de que o trabalho satisfaz as necessidades materiais e psicológicas.

O comportamento dos empregados em iniciativas organizacionais de RSC destaca uma motivação específica para participar e contribuir para essas atividades. Como resultado, o processo de envolvimento em atividades de RSC deve ter um efeito recíproco no nível geral de motivação dos empregados (Mozes, Josman, \& Yaniv, 2011).

Um ambiente de trabalho bem projetado também pode ajudar a alcançar a satisfação profissional dos empregados, e pode aumentar a produtividade e a qualidade do trabalho quando o ambiente é desejável para eles. Um tratamento justo e igual é também um fator, porque o tratamento igual pode levá-los a ter atitudes de trabalho positivas, e isso resultará na satisfação no trabalho (Aban, Perez, Ricarte, \& Chiu, 2019).

A satisfação dos empregados com o trabalho desempenha um papel fundamental na determinação do sucesso e do fracasso de empresas. Assim, as organizações têm se concentrado em melhorar a satisfação no trabalho de seus colaboradores para atingir metas e objetivos organizacionais (Priyanka et al., 2020).

A fim de garantir a prevalência da RSC, uma organização deve manter o bom relacionamento com seus empregados. Se uma organização não assume a responsabilidade para com seus empregados, não pode fazê-lo com seus clientes ou em relação ao ambiente em que opera (Asrar-ul-Haq et al., 2017).

Ainda, com a finalidade de formar a atratividade da organização, percebida por funcionários em potencial, os valores percebidos, a ética e as capacidades de resposta social da organização desempenham um papel importante (Greening \& Turban, 2000).

A percepção de que a organização mostra comportamentos justos e honestos no ambiente externo tem um impacto importante nas atitudes dos empregados, como satisfação no trabalho e comprometimento organizacional (Boğan et al., 2018; Rupp, Ganapathi, Aguilera, \& Williams, 2006).

Organizar e participar de atividades de RSC pode melhorar a imagem da organização na mente dos funcionários e aumentar sua satisfação (Asrar-ul-Haq et al., 2017). Portanto, pode-se supor que:

H3 - A responsabilidade social percebida impacta a satisfação do trabalhador.

A experiência de um empregado com significância sobre o trabalho amplia seu desejo de buscar ativamente informações e experiências úteis para sua função, e esse desejo, por sua vez, cria apego ao trabalho (Han, Sung, \& Suh, 2021). Assim, os trabalhadores experimentam satisfação no emprego em parte porque o veem como significativo (Allan, Dexter, Kinsey, \& Parker, 2018).

Contando com uma amostra composta por 602 enfermeiros, empregados em dois hospitais de diferentes cidades no noroeste da Itália, Ghislieri et al. (2019) validaram a hipótese que indicava a relação direta e positiva entre a significância do trabalho e a satisfação do trabalhador. Nesse contexto, foi elaborada a seguinte hipótese:

H4 - A significância do trabalho impacta a satisfação do trabalhador.

\begin{tabular}{|l|l|l|l|l|l|}
\hline Rev. Gest. Soc. Ambient. & São Paulo (SP) & v.15 & p.01-19 & e02769 & 2021. \\
\hline
\end{tabular} 
Quando os empregados percebem que a organização os está apoiando, eles acreditam que ela está sendo justa e, portanto, respondem positivamente, por exemplo, por meio de uma maior satisfação no trabalho e maior compromisso organizacional (Rhoades \& Eisenberger, 2002).

Allen, Shore e Griffeth (2003) conduziram uma pesquisa com dois grupos. A amostra 1 era composta de 264 vendedores, que trabalhavam nas áreas de beleza e cosméticos de uma grande loja de departamentos no sudeste dos EUA. A amostra 2 contou com 345 corretores de seguros de uma grande seguradora nacional. Os resultados indicaram uma relação direta e positiva entre suporte organizacional percebido e satisfação do trabalhador nos dois momentos do estudo. Diante dos trabalhos apresentados, tem-se a seguinte hipótese:

\section{H5 - O suporte organizacional percebido impacta a satisfação do trabalhador.}

\subsection{Modelo Teórico}

A base teórica desta pesquisa é desenvolvida a partir das evidências empíricas e dos pressupostos de estudos anteriores. Esses estudos destacaram a relação da RSC percebida com a satisfação no trabalho, seja diretamente, seja mediada pela significância do trabalho e pelo suporte organizacional percebido.

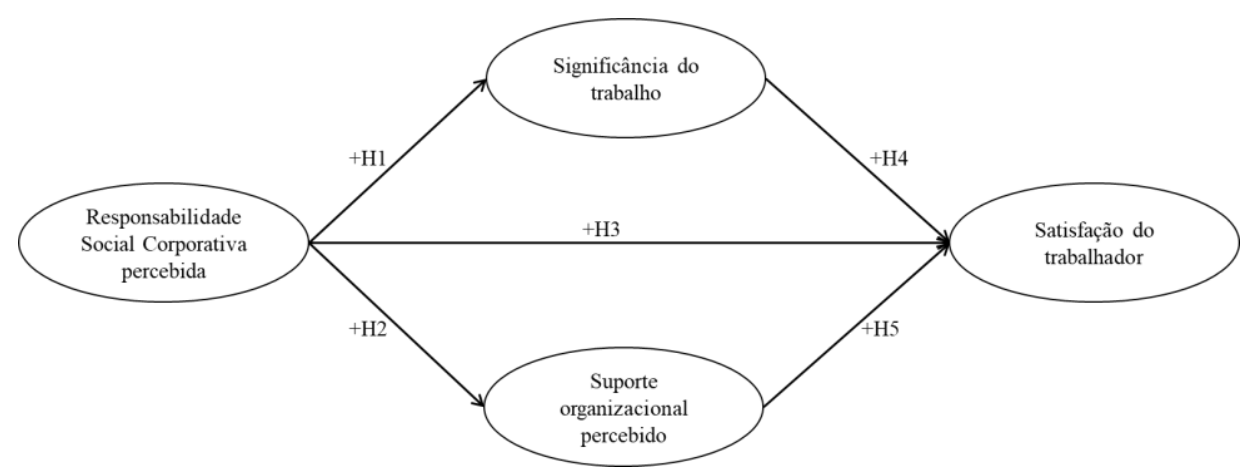

Figura 1 - Modelo teórico da pesquisa

Fonte: Elaborado pelos autores (2021).

Diante disso, conforme foi demonstrado na Figura 1, o modelo teórico foi construído conforme segue: (1) RSC percebida (variável independente); (2) significância do trabalho (variável independente, mediadora); (3) suporte organizacional percebido (variável independente, mediadora); (4) satisfação no trabalho (variável dependente).

\section{MÉTODOS}

Esta pesquisa está dividida em duas etapas. A primeira se refere à tradução, adaptação, aplicação e validação da escala de responsabilidade social corporativa percebida, proposta por Glavas e Kelley (2014). Na segunda etapa são incluídas questões sobre satisfação do trabalho, significância do trabalho e suporte organizacional percebido, que visam a atender ao objetivo da pesquisa. Ressalta-se que a utilização do instrumento foi autorizada pelos autores americanos.

\subsection{Validação da escala}

Essa etapa envolveu dois momentos: o primeiro consistiu em traduzir e adaptar os itens da escala. O documento foi traduzido por especialista em traduções inglês-português, 
sendo em seguida apresentado aos juízes, doutores com notório saber na área de RSC, que contribuíram com a adaptação semântica.

A segunda etapa envolveu a análise estatística das propriedades psicométricas e fatorial da versão em português da escala de responsabilidade social percebida.

\subsection{Amostra}

A amostra contou com 190 empregados da Universidade de Fortaleza, instituição privada de ensino superior, os quais responderam, de forma voluntária, a um questionário disponibilizado por intermédio da universidade, após a aprovação da pesquisa pelo Comitê de Ética em Pesquisa (Coética) da Universidade de Fortaleza - CAAE 39222220.1.0000.5052.

Para determinar o tamanho da amostra, foi utilizado o aplicativo $G$ *Power, versão 3.1, que estimou 68 casos, para um poder amostral de 0,80 e um efeito $\left(\mathrm{f}^{2}\right)$ de 0,15 . Hair, Black, Babin, Anderson e Tatham (2009) apresentam uma regra de duas a três vezes o valor calculado como amostra ideal, ou seja, entre 136 e 204. Portanto, a amostra atende ao requisito proposto.

\subsection{Instrumento de pesquisa}

Todos os participantes responderam a um questionário do tipo Likert, contendo a escala de percepção da responsabilidade social e questões envolvendo satisfação do trabalho, significância do trabalho, suporte organizacional percebido e satisfação do trabalhador com respostas variando de discordo totalmente a concordo totalmente, além de questões sociodemográficas sobre idade, gênero, tempo trabalhando na IES e nível de escolaridade que caracterizam a amostra.

Para fins de verificação da consistência interna do instrumento, foi calculado o alfa de Cronbach $(\alpha)$, com valor sugerido igual ou acima de 0,70 para que o índice seja considerado confiável (Hair, Sarstedt, Hopkins, \& Kuppelwieser, 2014).

Como forma de controle do viés do métod o comum, foram adotados os procedimentos de garantia do anonimato dos respondentes e foi informado que não há respostas certas ou erradas (Pod sakoff, MacKenzie, Lee, \& Pod sakoff, 2003).

A Figura 2 apresenta as questões envolvidas na pesquisa.

\begin{tabular}{|l|l|l|}
\hline Construto & Questão & Fonte \\
\hline $\begin{array}{l}\text { RSC percebida } \\
\text { RSC })\end{array}$ & $\begin{array}{l}\text { Contribuir para o bem-estardos empregadosé uma alta prioridade na } \\
\text { organização em que trabalho. } \\
\text { Contribuir para o bem-estar dos clientes é uma alta prioridade na } \\
\text { organização em que trabalho. } \\
\text { Contribuir para o bem-estar dos fornecedores é uma alta prioridade } \\
\text { na organização em que trabalho. } \\
\text { Contribuir para o bem-estarda comunidade é uma alta prioridade na } \\
\text { organização em que trabalho. } \\
\text { As questões a mbientais são parte integrante da estratégia da } \\
\text { organização em que trabalho. } \\
\text { Tratar de questões ambientais é parte integrante das operações } \\
\text { diárias da organização em que trabalho. } \\
\text { A organização em que trabalho cuida bastante para que nosso } \\
\text { trabalho não prejudique o meio ambiente. } \\
\text { A organização em que trabalho atinge suas metas de curto prazo, } \\
\text { enquanto se mantém focada em seu impacto sobre o meio ambiente. }\end{array}$ & \\
\hline
\end{tabular}




\begin{tabular}{|c|c|c|}
\hline $\begin{array}{l}\text { Suporte } \\
\text { organizacional } \\
\text { percebido (SOP) } \\
\alpha=0,958\end{array}$ & $\begin{array}{l}\text { Minha organização valoriza as contribuições para o bem-estar dela. } \\
\text { Minha organização realmente se preocupa com o bem-estar dos } \\
\text { empregados. } \\
\text { Minha organização considera fortemente as metas e os valores dos } \\
\text { empregados. } \\
\text { Minha organização está disposta a ajudar os empregados se eles } \\
\text { precisarem de um fa vor especial. } \\
\text { Minha organização mostra muita preocupação com os empregados. } \\
\text { Minha organização se orgulha das realizações dos empregados no } \\
\text { trabalho. }\end{array}$ & $\begin{array}{l}\text { Eisenberger et } \\
\text { al. (1997) }\end{array}$ \\
\hline $\begin{array}{l}\text { Significância do } \\
\text { trabalho (SIG) } \\
\alpha=0,912\end{array}$ & $\begin{array}{l}\text { O trabalho que faço é muito importante para mim. } \\
\text { Minhas atividades profissionais são pessoalmente significativas para } \\
\text { mim. } \\
\text { O trabalho que faço é significativo para mim. }\end{array}$ & $\begin{array}{l}\text { Spreitzer } \\
(1995)\end{array}$ \\
\hline $\begin{array}{l}\text { Satisfação do } \\
\text { trabalhador(ST) } \\
\alpha=0,913\end{array}$ & $\begin{array}{l}\text { No total, estou satisfeito com meu trabalho. } \\
\text { Em geral, gosto do meu trabalho. } \\
\text { Em geral, eu gosto de trabalhar aqui. }\end{array}$ & $\begin{array}{l}\text { Cammann et al. } \\
\text { (1983) }\end{array}$ \\
\hline
\end{tabular}

Figura 2 - Instrumento de pesquisa

Fonte: Elaborado pelos autores (2021).

\subsection{Análise dos dados}

Os dados foram extraídos do formulário eletrônico e transportados para serem tratados no aplicativo SPSS® da IBM® versão 20, e para gerar a estatística descritiva.

Com relação ao tratamento dos dados, pelo fato de a coleta ter ocorrido de forma eletrônica e com respostas obrigatórias, não foram encontrados dados faltantes. Por decisão dos autores, os dados discrepantes foram mantidos como forma de preservar a real intenção de cada respondente.

As respostas foram medidas seguindo uma escala Likert de cinco pontos $(1=$ discordo totalmente a $7=$ concordo totalmente). Os dados foram analisados por meio de estatística descritiva e inferencial. Testes de confiabilidade e de hipóteses também foram realizados para medir a autenticação dos dados. Para a verificação das hipóteses do estudo de pesquisa, a modelagem de equações estruturais (SEM) foi aplicada por meio do software SmartPLS versão 3.3.3, que é uma das principais ferramentas para equações estruturais de modelação pelo método mínimos quadrados parciais (PLS-SEM) (Ringle, Wende, \& Becker, 2015).

\section{RESULTADOS E DISCUSSÕES}

Nesta seção são apresentados os resultados da pesquisa, iniciando com a validação da pesquisa, análises descritivas e confirmação das hipóteses.

\subsection{Validação da escala de RSC percebida}

Foram efetuados testes de homogeneidade das variâncias (teste de Levene) e de igualdade das médias (teste $\mathrm{t}$ ) com a finalidade de comprovar que os respondentes pertencem à mesma amostra.

Conforme demonstrado na Tabela 1 , os dois testes apresentaram valores de $\mathrm{p}>0,05$. Nesse caso, deixam de rejeitar a hipótese nula, podendo-se afirmar com 95\% de probabilidade 
que há homogeneidade das variâncias e que as médias das variáveis são iguais. Dessa forma, a amostra pertence ao mesmo grupo de respondentes (Fávero \& Belfiore, 2020).

Tabela 1 - Testes de homogeneidade das variâncias e de igualda de das médias

\begin{tabular}{lccccc}
\hline Item & \multicolumn{2}{c}{ Teste de Levene } & \multicolumn{3}{c}{ Teste t } \\
\hline & Estatística $\mathbf{F}$ & Valor de $\mathbf{p}$ & Valor de t & gl & Valor de p \\
\hline RSC1 & 0,005 & 0,945 & 0,841 & 188 & 0,401 \\
\hline RSC2 & 1,213 & 0,272 & $-0,397$ & 188 & 0,692 \\
\hline RSC3 & 0,446 & 0,505 & $-0,315$ & 188 & 0,753 \\
\hline RSC4 & 0,329 & 0,567 & $-0,210$ & 188 & 0,834 \\
\hline RSC5 & 0,089 & 0,766 & 0,292 & 188 & 0,770 \\
\hline RSC6 & 0,285 & 0,594 & $-0,044$ & 188 & 0,965 \\
\hline RSC7 & 0,695 & 0,405 & 0,606 & 188 & 0,545 \\
\hline RSC8 & 1,516 & 0,220 & 1,094 & 188 & 0,276 \\
\hline
\end{tabular}

Fonte: Elaborado pelos autores (2021).

A Tabela 2 demonstra a correlação entre as variáveis da escala de responsabilidade social corporativa percebida.

Conforme apresentado na Tabela 2, os valores do coeficiente de variação foram inferiores a $30 \%$, demonstrando que a amostra tem certa homogeneidade (Fávero \& Belfiore, 2020). As correlações são consideradas fracas quando $r=0,10$ até 0,30 ; moderada quando $r=$ 0,40 até 0,60; e forte quando $\mathrm{r}=0,70$ até 1 (Dancey \& Reidy, 2008). Conforme observado, as correlações são consideradas de moderadas a fortes.

Tabela 2 - Média, desvio-padrão, coeficiente de variação e correlação entre os itens

\begin{tabular}{|c|c|c|c|c|c|c|c|c|c|c|c|}
\hline Item & $\overline{\mathbf{M}}$ & $\overline{D P}$ & $\overline{C V}$ & 1 & 2 & 3 & 4 & 5 & 6 & 7 & $\overline{8}$ \\
\hline RSC1 & 5,39 & 1,432 & $26,6 \%$ & 1,000 & & & & & & & \\
\hline RSC2 & 5,95 & 1,128 & $19,0 \%$ & $0,677^{*}$ & 1,000 & & & & & & \\
\hline RSC3 & 5,31 & 1,286 & $24,2 \%$ & $0,689^{*}$ & $0,670^{*}$ & 1,000 & & & & & \\
\hline RSC4 & 5,73 & 1,194 & $20,8 \%$ & $0,747^{*}$ & $0,792^{*}$ & $0,716^{*}$ & 1,000 & & & & \\
\hline RSC5 & 5,74 & 1,200 & $20,9 \%$ & $0,678^{*}$ & $0,702^{*}$ & $0,669 *$ & $0,793^{*}$ & 1,000 & & & \\
\hline RSC6 & 5,59 & 1,208 & $21,6 \%$ & $0,674^{*}$ & $0,661^{*}$ & $0,691 *$ & $0,794^{*}$ & $0,916^{*}$ & 1,000 & & \\
\hline RSC7 & 5,61 & 1,283 & $22,9 \%$ & $0,709^{*}$ & $0,660^{*}$ & $0,686^{*}$ & $0,757^{*}$ & $0,865^{*}$ & $0,881^{*}$ & 1,000 & \\
\hline RSC8 & 5,48 & 1,371 & $25,0 \%$ & $0,696^{*}$ & $0,579^{*}$ & $0,641 *$ & $0,709 *$ & $0,850 *$ & $0,856^{*}$ & $0,873^{*}$ & 1,000 \\
\hline
\end{tabular}

Nota: * Correlação significante a $1 \%$, bicaudal.

Fonte: Elaborado pelos autores (2021).

Foi efetuada a análise fatorial por componentes principais, o que permite, a partir de um conjunto de variáveis originais, que sejam gerados fatores que representam o comportamento conjunto das variáveis (Fávero \& Belfiore, 2020). O resultado apresentou estatística $\mathrm{KMO}=0,926$ (quanto mais próximo de um, melhor), e teste de esfericidade de Bartlett significante $(\mathrm{p}<0,001)$ (Hair et al., 2009). As variáveis foram agrupadas em apenas um fator com $77,17 \%$ do total da variância explicada. A Tabela 3 demonstra as cargas fatoriais de cada item, que formam a escala de responsabilidade social corporativa percebida. 
Tabela 3 - Análise fatorial

\begin{tabular}{ccc}
\hline Item & Carga fatorial & Comunalidades \\
\hline RSC1 & 0,832 & 0,692 \\
\hline RSC2 & 0,813 & 0,660 \\
\hline RSC3 & 0,816 & 0,665 \\
\hline RSC4 & 0,898 & 0,806 \\
\hline RSC5 & 0,926 & 0,858 \\
\hline RSC6 & 0,926 & 0,858 \\
\hline
\end{tabular}

Fonte: Elaborado pelos autores (2021).

Conforme demonstrado na Tabela 3, os valores das cargas fatoriais são considerados adequados por estarem acima de 0,70 , e os valores das comunalidades são superiores ao valor mínimo exigido de 0,50 (Hair et al., 2009).

Considerando que a análise fatorial por componentes principais apresentou valores adequados para a escala de responsabilidade social corporativa percebida, entende-se que foi validada para utilização na língua portuguesa.

\subsection{Análise descritiva da pesquisa}

A Tabela 4 demonstra os resultados descritivos das questões sociodemográficas dos respondentes. Verifica-se a predominância do sexo feminino (56,3\%), da faixa etária entre 36 e 45 anos $(30,0 \%)$, com ensino médio $(33,2 \%)$ e com vínculo empregatício acima de 10 anos $(32,6 \%)$.

Tabela 4 - Dados sociodemográficos

\begin{tabular}{|c|c|c|c|}
\hline Variável & Item & N. ${ }^{\circ}$ & $\%$ \\
\hline \multirow[t]{2}{*}{ Sexo } & Masculino & 83 & 43,7 \\
\hline & Feminino & 107 & 56,3 \\
\hline \multirow[t]{5}{*}{ Faixa etária } & Até 25 anos & 22 & 11,6 \\
\hline & De 26 a 35 anos & 58 & 30,5 \\
\hline & De 36 a 45 anos & 57 & 30,0 \\
\hline & De 46 a 55 anos & 38 & 20,0 \\
\hline & Acima de 55 anos & 15 & 7,9 \\
\hline \multirow[t]{6}{*}{ Escolaridade } & Fundamental & 5 & 2,6 \\
\hline & Médio & 63 & 33,2 \\
\hline & Superior & 43 & 22,6 \\
\hline & Pós-graduado & 1 & 11,1 \\
\hline & Mestrado & 33 & 17,4 \\
\hline & Doutorado & 25 & 13,2 \\
\hline \multirow[t]{4}{*}{ Tempo de vínculo com a instituição } & Até 2 anos & 41 & 21,6 \\
\hline & Acima de 2 até 5 anos & 36 & 18,9 \\
\hline & Acima de 5 até 10 anos & 51 & 26,8 \\
\hline & Acima de 10 anos & 62 & 32,9 \\
\hline
\end{tabular}

Fonte: Elaborado pelos autores (2021).

\subsection{Modelagem de equações estruturais (MME)}

Conforme sugerido por Hair et al. (2014), foram utilizadas as cargas fatoriais, alfa de Cronbach, confiabilidade composta e variância média extraída para avaliar a validade de convergência. Para medir a confiabilidade ou a consistência interna, foi utilizado o alfa de Cronbach, medida que varia de zero a um, sendo o valor 0,70 o limite mínimo aceitável (Hair et al., 2009). Nesse caso, também se utiliza da confiabilidade composta para medir a 
consistência interna, por fornecer uma medida mais apropriada. O valor mínimo aceitável é 0,70 (Hair et al., 2014).

Em seguida, é verificada a validade dos construtos, que foram examinados observando-se as validades convergente e discriminante de um construto. O suporte é fornecido para validade convergente, quando cada item tem cargas externas acima de 0,70 e quando a variância média extraída de cada construto (AVE) é 0,50 ou superior. O AVE é o grande valor médio das cargas quadradas de um conjunto de indicadores (Hair et al., 2014) e é equivalente à comunalidade de um construto.

A validade discriminante representa até que ponto o construto é empiricamente distinto de outros construtos ou, em outras palavras, o construto mede o que se destina a medir. Um método para avaliar a existência de validade discriminante é o critério de Fornell e Larcker (1981). Esse método afirma que o construto compartilha mais variância com seus indicadores do que com qualquer outro construto. Para testar esse requisito, o AVE de cada construto deve ser maior do que a correlação quadrática mais alta com qualquer outro construto. Conforme demonstrado na Tabela 5, os valores das AVEs são superiores a 0,50, e o critério de Fornell e Larcker (1981) foi atendido (Henseler, Ringle, \& Sinkovics, 2009).

Os valores de VIF (multicolinearidade) ficaram dentro do esperado (Hair et al., 2014). Para isso, foi eliminado o item ST03 do construto significância do trabalho, por apresentar valor de VIF superior a 10.

Tabela 5 - Validade discriminante e validade convergente

\begin{tabular}{lcccc}
\hline & RSC & ST & SIG & SOP \\
\hline RSC percebida & 0,878 & & & \\
\hline Satisfação do trabalhador & 0,780 & 0,928 & & \\
\hline Significância do trabalho & 0,737 & 0,873 & 0,977 & 0,929 \\
\hline Suporte organizacionalpercebido & 0,786 & 0,773 & 0,687 & 0,968 \\
\hline \hline Alfa de Cronbach & 0,957 & 0,919 & 0,952 & 0,974 \\
\hline Confiabilidade composta & 0,964 & 0,949 & 0,977 & 0,864 \\
\hline Variância média extraída & 0,772 & 0,860 & 0,955 & 2,79 \\
\hline VIF & 3,26 & 3,09 & 2,34 & \\
\hline
\end{tabular}

Fonte: Elaborado pelos autores (2021).

Uma vez que a confiabilidade e a validade dos modelos externos são estabelecidas, a próxima etapa é a avaliação da qualidade do modelo, baseada em sua capacidade de prever os construtos endógenos. Os critérios utilizados formam o coeficiente de determinação $\left(\mathrm{R}^{2}\right)$ e a redundância validada cruzada $\left(\mathrm{Q}^{2}\right)$ (Henseler et al., 2009).

$\mathrm{O}$ coeficiente de determinação $\left(\mathrm{R}^{2}\right)$ é uma medida da precisão preditiva do modelo. Cohen (2013) estimou que $\mathrm{R}^{2}=2 \%=$ pequeno; $\mathrm{R}^{2}=13 \%=$ médio; $\mathrm{R}^{2}=26 \%=$ grande. $\mathrm{De}$ acordo com a Tabela 6 , os construtos apresentam valores de coeficientes de determinação (isto é, o percentual de variância explicada das variáveis endógenas) considerados grandes (Bido \& Silva, 2019).

Tabela 6 - Avaliação do modelo interno

\begin{tabular}{lccc}
\hline Constructo & $\mathbf{R}^{\mathbf{2}}$ & $\mathbf{R}^{\mathbf{2}}$ ajustado & $\mathbf{Q}^{\mathbf{2}}$ \\
\hline Satisfação do trabalhador & 0,826 & 0,824 & 0,700 \\
\hline Significância do trabalho & 0,544 & 0,541 & 0,512 \\
\hline Suporte organiza cionalpercebido & 0,618 & 0,615 & 0,526 \\
\hline Fon
\end{tabular}

Fonte: Elaborado pelos autores (2021).

O tamanho do efeito para cada modelo de caminho pode ser determinado calculando o $\mathrm{f}^{2}$ de Cohen. $\mathrm{O} \mathrm{f}^{2}$ é calculado observando a mudança em $\mathrm{R}^{2}$, quando um construto específico é 
eliminado do modelo. Quanto ao tamanho do efeito: $\mathrm{f}^{2}=0.02=$ pequeno; $\mathrm{f}^{2}=0.15=$ médio; $\mathrm{f}^{2}$ $=0.35=$ grande $($ Cohen, 2013).

Tabela 7 - Coeficientes estruturais

\begin{tabular}{lccccccccc}
\hline Relação & Hipótese & $\boldsymbol{\beta}$ & DP & IC 95\% & $\mathbf{f}^{\mathbf{2}}$ & $\mathbf{t}$ & \multicolumn{1}{c}{ p } & Suportada \\
\hline $\mathrm{RSC} \rightarrow$ SIG & H1 & 0,737 & 0,051 & 0,$620 ; 0,821$ & 1,19 & 14,472 & 0,000 & SIM \\
\hline $\mathrm{RSC} \rightarrow$ SOP & H2 & 0,786 & 0,037 & 0,$700 ; 0,845$ & 1,61 & 21,413 & 0,000 & SIM \\
\hline RSC $\rightarrow$ ST & H3 & 0,141 & 0,071 & 0,$018 ; 0,302$ & 0,04 & 1,983 & 0,055 & NÃO \\
\hline SIG $\rightarrow$ ST & H4 & 0,595 & 0,063 & 0,$477 ; 0,722$ & 0,87 & 9,495 & 0,000 & SIM \\
\hline
\end{tabular}

Fonte: Elaborado pelos autores (2021).

Conforme demonstrado na Tabela 7, a hipótese que sugeria a influência da responsabilidade social corporativa percebida na significância do trabalho (H1) foi suportada $(\beta=0,739, \mathrm{p}<0,001)$; o resultado está similar ao encontrado no trabalho de Nazir e Islam (2020), que validaram a hipótese $(\beta=0,670, \mathrm{p}<0,001)$, bem como à pesquisa de Afsar et al. (2019), que também validaram a hipótese $(b=0,670, p<0,001)$.

Como relação à hipótese $\mathrm{H} 2$, que indicava a relação entre RSC percebida e suporte organizacional percebido, esta foi confirmada $(\beta=0,786, \mathrm{p}<0,001)$, resultado análogo ao encontrado por Glavas e Kelley (2014) $(\beta=0,574$, p $<0,001)$, que validaram a relação entre RSC percebida e SOP, e à pesquisa de Ellis (2009), que confirmou a mesma hipótese ( $\beta=$ 0,247, $\mathrm{p}<0,05)$.

Também foi previsto que a responsabilidade social corporativa percebida influencia a satisfação do trabalhador. Conforme demonstrado na Tabela 7, a hipótese H3 não foi suportada $(\beta=0,141, \mathrm{p}>0,05)$, resultado semelhante ao do trabalho de Glavas e Kelley (2014) $(\beta=0,080, \mathrm{p}>0,05)$.

A hipótese $\mathrm{H} 4$, que previa a relação entre significância do trabalho e satisfação do trabalho, também foi suportada $(\beta=0,654, \mathrm{p}<0,001)$, resultado semelhante ao do trabalho de Glavas e Kelley (2014) $(\beta=0,463, \mathrm{p}<0,01)$, que confirmaram a mesma hipótese, e à pesquisa de Ghislieri et al. (2019), que também validaram a hipótese $(\beta=0,480, p<0,001)$. Dessa forma, entende-se que a significância é de natureza relacional, com os empregados descobrindo um senso de propósito superior, com base em como a organização trata os outros (Glavas \& Kelley, 2014).

No mesmo sentido, a hipótese $\mathrm{H} 5$, que apontava para a relação entre o suporte organizacional percebido e a satisfação do trabalhador, foi confirmada $(b=0,255, p<0,001)$, resultado similar ao encontrado por Glavas e Kelley (2014) $(\beta=0,364, p<0,05)$. A pesquisa de Aban et al. (2019) confirmou a mesma hipótese $(\beta=0,760, \mathrm{p}<0,01)$.

\subsection{Discussão dos resultados}

Especificamente, a ligação entre a RSC e a significância do trabalho pode ser explicada pelas perspectivas psicológica e sociológica. Do ponto de vista psicológico, a participação em práticas de RSC permite que os colaboradores percebam uma maior significância do trabalho, melhorando sua autoestima. Ao participarem de práticas de RSC, os membros podem perceber que são capazes não apenas de influenciar positivamente os outros ou a sociedade, mas também de fazer melhorias substanciais. Esse sentimento intensificado de impacto positivo os induzirá a acreditar em sua capacidade com confiança, aumentando sua autoestima (Kim et al., 2018b). 
Uma das maneiras de observar o papel da RSC na vida organizacional é verificar seu impacto na satisfação com o trabalho dos funcionários. A satisfação no trabalho é um estado emocional positivo que resulta da avaliação da experiência de trabalho de uma pessoa em relação ao seu trabalho, como resultado de suas percepções sobre este trabalho (Purba et al., 2019), ademais, o sucesso das ações realizadas por uma organização depende, em grande medida, da aceitação dessas ações por parte dos funcionários (Ribeiro, Puente-Palacios, \& Ferreira, 2015).

Nesse sentido, verifica-se que estudos anteriores confirmaram o impacto da RSC percebida na satisfação do trabalhador (Asrar-ul-Haq et al., 2017; Tziner, Oren, Bar, \& Kadosh, 2011; Valentine \& Fleischman, 2007). No entanto, o impacto esperado não foi encontrado em alguns estudos (Glavas \& Kelley, 2014). Esse caso traz à mente que poderia haver outras variáveis mediando a relação entre a RSC e a satisfação no trabalho. No presente estudo, constatou-se que a significância do trabalho e o suporte organizacional percebido afetaram a relação entre a responsabilidade social corporativa percebida e a satisfação no trabalho. Já a mediação com o suporte organizacional percebido apresentou um coeficiente maior do que a mediação pela significância.

O efeito positivo das percepções de RSC pode ser aumentado pela experiência edificante de suporte organizacional percebido de uma empresa, fortalecendo assim seu efeito positivo (Hur et al., 2019). Os empregados ficam mais satisfeitos com seu trabalho quando percebem que os superiores estão lhes dando o devido suporte (Aban et al., 2019).

A forte relação entre as percepções dos funcionários sobre a RSC e a significância do trabalho indica que benefícios importantes podem ser trazidos por meio do investimento em RSC, especialmente aquele direcionado às partes interessadas internas. Melhorias na RSC interna podem ser alcançadas através da melhoria do ambiente de trabalho para os funcionários, dando-lhes mais oportunidades de acesso à formação profissional e atendendo às suas necessidades individuais.

Em um contexto organizacional, a implementação de programas de RSC não apenas contribui para enriquecer a reputação e a identidade organizacional, mas também auxilia no aumento da satisfação profissional dos empregados, estabelecendo a sensação de trabalhar em uma organização socialmente responsável (Priyanka et al., 2020). Uma vez que as percepções e as atitudes de trabalho dos empregados têm uma enorme influência sobre o desempenho organizacional, a tentativa de melhorar as percepções e as atitudes de membros através de práticas de RSC é uma opção valiosa, na qual vale a pena investir (Kim et al., 2018a).

\subsection{Contribuições teóricas e gerenciais}

Como primeira contribuição, esta pesquisa se concentra nas percepções dos funcionários com relação à RSC da organização, conforme a sugestão de Valentine e Fleischman (2007) e Glavas e Godwin (2013), que mostraram a necessidade de estudar medidas subjetivas individuais de RSC. Com isso, em segund o lugar, este estudo aumenta a compreensão das consequências das percepções pelos funcionários sobre a RSC, notadamente com relação à satisfação do trabalhador, incluindo variáveis como o suporte organizacional percebido e a significância do trabalho.

Os resultados deste estudo também fornecem contribuições práticas, mostrando a importância das percepções dos funcionários. De fato, os resultados mostram que, para obter melhor satisfação dos funcionários, as empresas devem melhorar as percepções destes sobre a RSC, pois uma percepção positiva da RSC tem um efeito positivo no suporte organizacional percebido e na significância do trabalho, que levam a uma satisfação do trabalhador.

Por fim, os resultados desta pesquisa geram implicações efetivas para as organizações, ao demonstrar que as ações de responsabilidade social podem gerar benefícios relevantes 
internamente, além dos próprios benefícios gerados para a sociedade. Para a academia, este estudo contribui com a discussão da RSC sob a percepção do empregado, em uma visão micro, individualizada da responsabilidade social nas organizações.

\section{CONCLUSÃO}

O presente estudo explora o impacto intraorganizacional dos programas de RSC nas atitudes dos empregados em relação ao trabalho e à organização, em termos de suporte organizacional, significância e satisfação do trabalhador.

Ficou comprovado que a responsabilidade social corporativa percebida está relacionada à satisfação no trabalho e que essa relação é mediada pela significância do trabalho e pelo suporte organizacional percebido. Nesse caso, os achados indicam que a RSC percebida não influencia diretamente a satisfação do trabalhador. Pode-se entender a partir desse fato que, além das práticas de RSC, a organização precisa desenvolver políticas internas para que se tenha o efeito positivo que a percepção da RSC gera na satisfação dos funcionários.

Este estudo apresenta como limitações a dificuldade de determinar a causalidade entre os construtos, em função da utilização da abordagem transversal e, também, o fato de os dados coletados representarem apenas uma instituição, o que dificulta a generalização dos resultados.

Como estudos futuros, sugere-se a aplicação de estudos longitudinais, para possibilitar uma análise de causalidade, estudos com organizações diferentes, como forma de sustentar os resultados deste artigo, e também a utilização de variáveis moderadoras para testar as relações em diferentes níveis das organizações.

\section{AGRADECIMENTOS}

Agradecemos ao Prof. Dr. Minelle Enéas da Silva por sua valiosa contribuição.

\section{REFERÊNCIAS}

Aban, C. J. I., Perez, V. E. B., Ricarte, K. K. G., \& Chiu, J. L. (2019). The relationship of organizational commitment, job satisfaction, and perceived organizational support of telecommuters in the national capital region. Review of Integrative Business and Economics Research, 8, 162-197.

Afsar, B., Al-Ghazali, B., \& Umrani, W. (2020). Retracted: Corporate social responsibility, work meaningfulness, and employee engagement: the joint moderating effects of incremental moral belief and moral identity centrality. Corporate Social Responsibility and Environmental Management, 27(3), 1264-1278. https://doi.org/10.1002/csr.1882

Aguinis, H. (2011). Organizational responsibility: Doing good and doing well. In S. Zedeck (Ed.), APA handbook of industrial and organizational psychology (Vol. 3, pp. 855-879). Washington, DC: American Psychological Association. https://doi.org/10.1037/12171-024

Aguinis, H., \& Glavas, A. (2019). On corporate social responsibility, sensemaking, and the search for meaningfulness through work. Journal of Management, 45(3), 1057-1086. 
Ahmad, R., Islam, T., \& Saleem, S. S. (2017). Employee engagement, organizational commitment and job satisfaction as consequent of perceived CSR: A mediation model. Journal of Research Society of Pakistan, 55(2), 153-168.

Ahmad, S., Shafique, O., \& Jamal, W. N. (2020). Impact of perceived corporate social responsibility on banks' financial performance and the mediating role of employees' satisfaction and loyalty in Pakistan. Journal of Accounting and Finance in Emerging Economies, 6(3), 765774. https://doi.org/10.26710/jafee.v6i3.1361

Allan, B. A., Dexter, C., Kinsey, R., \& Parker, S. (2018). Meaningful work and mental health: Job satisfaction as a moderator. Journal of Mental Health, 27(1), 38-44. https://doi.org/10.1080/09638237.2016.1244718

Allen, D. G., Shore, L. M., \& Griffeth, R. W. (2003). The role of perceived organizational support and supportive human resource practices in the turnover process. Journal of Management, 29(1), 99-118. https://doi.org/10.1177/014920630302900107

Asrar-ul-Haq, M., Kuchinke, K. P., \& Iqbal, A. (2017). The relationship between corporate social responsibility, job satisfaction, and organizational commitment: Case of Pakistani higher education. Journal of Cleaner Production, 142, 2352-2363.

Azim, M. T. (2016). CSR and employee behavior: Mediating role of organizational commitment. Revista Brasileira de Gestão de Negócios, 18(60), 207-225. https://doi.org/10.7819/rbgn.v18i60.2319

Aziri, B. (2011). Job satisfaction: A literature review. Management Research and Practice, 3(4), $77-86$.

Bido, D. de S., \& Silva, D. (2019). SmartPLS 3: Especificação, estimação, avaliação e relato. $\begin{array}{lllll}\text { Administração: } \quad \text { Ensino } & e & \text { Pesquisa, } & \text { 20(2), }\end{array}$ https://doi.org/10.13058/raep.2019.v20n2.1545

Boğan, E., Türkay, O., \& Dedeoğlu, B. B. (2018). Perceived corporate social responsibility and job satisfaction: The mediator role of organizational identification. International Journal of Business and Management Studies, 10(2), 51-67

Carroll, A. B. (1979). A three-dimensional conceptual model of corporate performance. Academy of Management Review, 4(4), 497-505. https://doi.org/10.5465/amr.1979.4498296

Carroll, A. B. (1999) Corporate social responsibility: Evolution of a definitional construct. Business \& Society, 38, 268-295. https://doi.org/10.1177/000765039903800303

Carroll, A. B. (2016). Carroll's pyramid of CSR: Taking another look. International Journal of Corporate Social Responsibility, 1(1), 3. https://doi.org/10.1186/s40991-016-0004-6

Chaudhary, R. (2019). Corporate social responsibility perceptions and employee engagement: Role of psychological meaningfulness, safety and availability. Corporate Governance: The International Journal of Business in Society, 19(4), 631-647. https://doi.org/10.1108/CG-062018-0207 
Chaudhary, R., \& Akhouri, A. (2019). CSR perceptions and employee creativity: Examining serial mediation effects of meaningfulness and work engagement. Social Responsibility Journal, 15(1), 61-74. https://doi.org/10.1108/SRJ-01-2018-0018

Cohen, J. (2013). Statistical power analysis for the behavioral sciences. Academic press.

Eisenberger, R., Huntington, R., Hutchison, S., \& Sowa, D. (1986). Perceived organizational support. Journal of Applied Psychology, 71(3), 500-507.

Eisenberger, R., Malone, G. P., \& Presson, W. D. (2016). Optimizing perceived organizational support to enhance employee engagement. Society for Human Resource Management and Society for Industrial and Organizational Psychology, 2, 1-22.

Ellis, A. D. (2009). The impact of corporate social responsibility on employee attitudes and behaviors. Academy of Management Proceedings, 1, 1-6). https://doi.org/10.5465/ambpp.2009.44251836

Fávero, L. P., \& Belfiore, P. (2020). Manual de análise de dados. Rio de Janeiro: LTC.

Fornell, C. G., \& Larcker, D. F. (1981), Evaluating structural equation models with unobservable variables and measurement error. Journal of Marketing Research, 18(1), 39-50. https://doi.org/10.1177/002224378101800104

Ghislieri, C., Cortese, C. G., Molino, M., \& Gatti, P. (2019). The relationships of meaningful work and narcissistic leadership with nurses' job satisfaction. Journal of Nursing Management, 27(8), 1691-1699. https://doi.org/10.1111/jonm.12859

Glavas, A., \& Godwin, L. N. (2013). Is the perception of 'goodness' good enough? Exploring the relationship between perceived corporate social responsibility and employee organizational identification. Journal of business ethics, 114(1), 15-27. https://doi.org/10.1007/s10551-012$\underline{1323-5}$

Glavas, A., \& Kelley, K. (2014). The effects of perceived corporate social responsibility on employee attitudes. Business Ethics Quarterly, 24(2), 165-202. https://doi.org/10.5840/beq20143206

Grant, A. M. (2008). Does intrinsic motivation fuel the prosocial fire? Motivational synergy in predicting persistence, performance, and productivity. Journal of Applied Psychology, 93(1), 4858. https://doi.org/10.1037/0021-9010.93.1.48

Greening, D. W., \& Turban, D. B. (2000). Corporate social performance as a competitive advantage in attracting a quality workforce. Business \& Society, 39(3), 254-280. https://doi.org/10.1177/000765030003900302

Hair, J. F., Black, W. C., Babin, B. J., Anderson, R. E., \& Tatham, R. L. (2009). Análise multivariada de dados. Bookman.

Hair, J. F. Jr., Sarstedt, M., Hopkins, L., \& Kuppelwieser, V. G. (2014). Partial least squares structural equation modeling (PLS-SEM). European Business Review, 26(2), 106-121. https://doi.org/10.1108/EBR-10-2013-0128 
Han, S.-H., Sung, M., \& Suh, B. (2021). Linking meaningfulness to work outcomes through job characteristics and work engagement. Human Resource Development International, 24(1), 3-22. https://doi.org/10.1080/13678868.2020.1744999

Henseler, J., Ringle, C. M., \& Sinkovics, R. R. (2009). The use of partial least squares path modeling in international marketing. New Challenges to International Marketing, 20, 277-319. https://doi.org/10.1108/S1474-7979(2009)0000020014

Hur, W. M., Moon, T. W., \& Choi, W. H. (2021). The role of job crafting and perceived organizational support in the link between employees' CSR perceptions and job performance: a moderated mediation model. Current Psychology, 40, 3151-3165. https://doi.org/10.1007/s 12144$\underline{019-00242-9}$

Khan, M., Sarwar, S., \& Khan, H. (2018). Impact of corporate social responsibility on job attitudes: Job satisfaction and organizational commitment of banking sector employees of Pakistan. SEISENSE Journal of Management, 1(3), 28-47. https://doi.org/10.33215/sjom.v1i3.22

Kim, B. J., Chang, Y. K., \& Kim, T. H. (2018a). How does corporate social responsibility promote innovation? The sequential mediating mechanism of employees' meaningfulness of work and intrinsic motivation. Proceedings of the $51^{\text {st }}$ Hawaii International Conference on System Sciences, p. 104-113.

Kim, B. J., Nurunnabi, M., Kim, T. H., \& Jung, S. Y. (2018b). The influence of corporate social responsibility on organizational commitment: The sequential mediating effect of meaningfulness of work and perceived organizational support. Sustainability, 10(7), 2208.

Kurtessis, J. N., Eisenberger, R., Ford, M. T., Buffardi, L., Stewart, K. A., \& Adis, C. S. (2015). Perceived organizational support: A meta-analytic evaluation of organizational support theory. Journal of Management, 43(6), 1854-1884. https://doi.org/10.1177/0149206315575554

Lima, M. X. A., Ferreira Neto, M. N., \& Pompeu, R. M. (2020). Projeto de extensão no ensino superior como prática de responsabilidade social. Revista de Gestão e Avaliação Educacional, 9(18), 1-12. https://doi.org/10.5902/2318133839368

Mozes, M., Josman, Z., \& Yaniv, E. (2011). Corporate social responsibility organizational identification and motivation. Social Responsibility Journal, 7(2), 310-325. https://doi.org/10.1108/17471111111141558

Nazir, O., \& Islam, J. U. (2020). Effect of CSR activities on meaningfulness, compassion, and employee engagement: A sense-making theoretical approach. International Journal of Hospitality Management, 90, 102630. https://doi.org/10.1016/j.ijhm.2020.102630

Podsakoff, P. M., MacKenzie, S. B., Lee, J.-Y., \& Podsakoff, N. P. (2003). Common method biases in behavioral research: A critical review of the literature and recommended remedies. Journal of Applied Psychology, 88(5), 879-903. https://doi.org/10.1037/0021-9010.88.5.879

Priyanka, P., Thevanes, N., \& Arulrajah, A. A. (2020). The impact of perceived corporate social responsibility on job satisfaction and organizational citizenship behavior in Sri Lanka Telecom. IUP Journal of Organizational Behavior, 19(2), 55-71. 
Purba, S. D., Chaterine, C., Hardjono, S., \& Clarissa, B. (2019). Psychological meaningfulness and work engagement effect on doctor's job satisfaction. JDM - Jurnal Dinamika Manajemen, 10(2), 229-239.

Ribeiro, P. E. da C. D., Puente-Palacios, K. E., \& Ferreira, T. V. A. (2015). Responsabilidade Socioambiental Nas Organizações: Uma Medida de Práticas Organizacionais e Endosso dos Trabalhadores. Revista de Gestão Social e Ambiental, 9(1), 36-50. https://doi.org/10.24857/rgsa.v9i1.947

Rhoades, L., \& Eisenberger, R. (2002). Perceived organizational support: A review of the literature. Journal of Applied Psychology, 87, 698-714.

Rowley, T., \& Berman, S. A. (2000). Brand new brand of corporate social performance. Business \& Society, 39, 397-418.

Rupp, D. E., Ganapathi, J., Aguilera, R. V., \& Williams, C. A. (2006). Employee reactions to corporate social responsibility: An organizational justice framework. Journal of Organizational Behavior: The International Journal of Industrial, Occupational and Organizational Psychology and Behavior, 27(4), 537-543. https://doi.org/10.1002/job.380

Shaw, W. S., Reme, S. E., Pransky, G., Woiszwillo, M. J., Steenstra, I. A., \& Linton, S. J. (2013). The pain recovery inventory of concerns and expectations: A psychosocial screening instrument to identify intervention needs among patients at elevated risk of back disability. Journal of Occupational and Environmental Medicine, 55, 885-894.

Spreitzer, G. M. (1995). Psychological empowerment in the workplace: Dimensions, measurement, and validation. Academy of Management Journal, 38: 1442-1465.

Tamm, K., Eamets, R., \& Mõtsmees, P. (2010). Relationship between corporate social responsibility and job satisfaction: The case of Baltic countries (SSRN Scholarly Paper No ID 1717710). Recuperado de https://papers.ssrn.com/abstract=1717710

Tziner, A., Oren, L., Bar, Y., \& Kadosh, G. (2011). Corporate social responsibility, organizational justice and job satisfaction: How do they interrelate, if at all? Journal of Work and Organizational Psychology, 27(1), 67-72.

Wang, Y. (2018). Perception of CSR and its consequences: A literature review. In $3^{\text {rd }}$ International Conference on Judicial, Administrative and Humanitarian Problems of State Structures and Economic Subjects (JAHP 2018). Atlantis Press. https://doi.org/10.2991/jahp$\underline{18.2018 .66}$

Valentine, S., \& Fleischman, G. (2007). Ethics programs, perceived corporate social responsibility and job satisfaction. Journal of Business Ethics, 77(2), 159-172. 